ANNALES

POLONICI MATHEMATICI

91.2-3 (2007)

\title{
A new invariant Kähler metric on relatively compact domains in a complex manifold
}

\author{
by Bo-Yong Chen (Shanghai)
}

\begin{abstract}
We introduce a new invariant Kähler metric on relatively compact domains in a complex manifold, which is the Bergman metric of the $L^{2}$ space of holomorphic sections of the pluricanonical bundle equipped with the Hermitian metric introduced by Narasimhan-Simha.
\end{abstract}

1. Introduction. It is well-known that for a bounded domain in $\mathbb{C}^{n}$, there are three canonical invariant metrics, i.e., the Carathéodory, Bergman and Kobayashi metrics (for their definitions and basic properties, see [6]). Moreover, if the domain is pseudoconvex, then there exists a complete invariant Kähler-Einstein metric (cf. [1], [10]). For a relatively compact domain in a complex manifold, these metrics might be degenerate (consider $\mathbb{C}^{n}$ and its compactification $\left.\mathbb{P}^{n+1}\right)$. It is also known that the canonical line bundle is closely related to the Bergman and Kähler-Einstein metrics.

In this paper, we introduce an invariant Kähler metric on complex manifolds with positive canonical bundle, which is in fact the Bergman metric with respect to a certain weighted Bergman space. Generally, the Bergman metric with respect to a weighted Bergman space is not invariant under the group of holomorphic transformations. Here we use a special weight originally contained in the work of Narasimhan-Simha [11]. Equipped with this weight, the associated Bergman metric turns out to be invariant.

We focus on the special case of relatively compact domains in complex manifolds. We give a localization principle for the new invariant metric on locally pseudoconvex domains in complex manifolds with positive canonical bundle; consequently, one can reduce the study to the case of bounded domains of holomorphy in $\mathbb{C}^{n}$. We also give a characterization for the exis-

2000 Mathematics Subject Classification: Primary 32F45; Secondary 32A25.

Key words and phrases: invariant metric, Kähler metric, canonical bundle.

Supported by grants 05QMX1452, NCET-05-0380 and Chinese Excellent Doctorate's Degree Thesis No. 200519; partially supported by NSFC No. 10571135 . 
tence of this invariant metric on locally pseudoconvex domains in complex manifolds with trivial canonical bundle.

This metric has the advantage of flexibility as regards completeness compared to the standard Bergman metric and is easier to analyze than the Kähler-Einstein metric. In fact, we conjecture that it is complete for any locally pseudoconvex domains in complex manifolds with positive canonical bundle. Although we cannot prove it, we provide a powerful evidence to support this conjecture by showing the completeness of the metric in the "worst" case, i.e., the complement of an effective divisor in a compact complex manifold with ample canonical bundle. It is well-known that if in addition the divisor is ample, then its complement carries a complete (invariant) Kähler-Einstein metric (cf. [7]). A little surprise is that the above two metrics are not equivalent.

The paper is organized as follows: In Section 2, we give the definition of the invariant metric and study its basic properties along the lines of [8]. In Section 3, we give a localization principle for this metric. In Section 4, we study the case of relatively compact Stein domains in a complex manifold with trivial canonical bundle. The last section investigates the asymptotic behavior of the metric on a hypersurface complement in a compact complex manifold with ample canonical bundle.

2. Definition and basic properties. Let $M$ be a complex manifold of dimension $n$ and $K_{M}$ its canonical line bundle. Let $\Gamma\left(M, m K_{M}\right)$ denote the space of holomorphic sections of $K_{M}^{\otimes m}$. For every integer $m \geq 1$, we define a continuous pseudonorm $\|\cdot\|_{m}$ on $\Gamma\left(M, m K_{M}\right)$ by

$$
\|s\|_{m}=\left\{\int_{M}\left[(-1)^{m n^{2} / 2} s \otimes \bar{s}\right]^{1 / m}\right\}^{m / 2}, \quad s \in \Gamma\left(M, m K_{M}\right) .
$$

Consider a volume form $\tau_{m}$ on $M$ defined by

$$
\tau_{m}=\sup _{\|s\|_{m}=1}\left[(-1)^{m n^{2} / 2} s \otimes \bar{s}\right]^{1 / m}
$$

Clearly $1 / \tau_{m}$ is a non-negative Hermitian metric on $K_{M}$ if $\tau_{m}$ is not identically zero. This metric was introduced by Narasimhan-Simha [11] in order to study the moduli space of compact complex manifolds with ample canonical bundle (for recent applications in complex geometry see Tsuji [14]).

LEMmA 2.1. The form $\tau_{m}$ is invariant under biholomorphic mappings.

Proof. Let $f: M \rightarrow N$ be a biholomorphic mapping. For any $s \in$ $\Gamma\left(N, m K_{N}\right)$, we have $\left\|f^{*} s\right\|_{m}=\|s\|_{m}$ by the transformation formula for integrals. Given $p \in M$, take a sequence $\left\{s_{k}\right\}$ in $\Gamma\left(N, m K_{N}\right)$ such that 
$\left\|s_{k}\right\|_{m}=1$ and

$$
\tau_{m}(f(p))=\lim _{k \rightarrow \infty}\left[(-1)^{m n^{2} / 2} s_{k}(f(p)) \otimes \bar{s}_{k}(f(p))\right]^{1 / m} .
$$

Then

$$
\begin{aligned}
f^{*} \tau_{m}(p) & =\tau_{m}(f(p))=\lim _{k \rightarrow \infty}\left[(-1)^{m n^{2} / 2} s_{k}(f(p)) \otimes \bar{s}_{k}(f(p))\right]^{1 / m} \\
& =\lim _{k \rightarrow \infty}\left[(-1)^{m n^{2} / 2} f^{*} s_{k}(p) \otimes \overline{f^{*} s_{k}}(p)\right]^{1 / m} \leq \tau_{m}(p) .
\end{aligned}
$$

The opposite inequality can be obtained similarly.

LEMMA 2.2. Let $M$ and $M^{\prime}$ be complex manifolds of complex dimensions $n$ and $n^{\prime}$ respectively. Then

$$
\tau_{m}\left(\left(p, p^{\prime}\right), M \times M^{\prime}\right)=\tau_{m}(p, M) \otimes \tau_{m}\left(p^{\prime}, M^{\prime}\right) .
$$

Proof. Take $\left\{s_{k}\right\} \subset \Gamma\left(M, m K_{M}\right)$ and $\left\{s_{k}^{\prime}\right\} \subset \Gamma\left(M^{\prime}, m K_{M^{\prime}}\right)$ such that $\left\|s_{k}\right\|_{m}=\left\|s_{k}^{\prime}\right\|_{m}=1$ and

$$
\begin{aligned}
\tau_{m}(p, M) & =\lim _{k \rightarrow \infty}\left[(-1)^{m n^{2} / 2} s_{k}(p) \otimes \bar{s}_{k}(p)\right]^{1 / m}, \\
\tau_{m}\left(p, M^{\prime}\right) & =\lim _{k \rightarrow \infty}\left[(-1)^{m n^{\prime 2} / 2} s_{k}^{\prime}(p) \otimes \overline{s_{k}^{\prime}}(p)\right]^{1 / m} .
\end{aligned}
$$

Fubini's theorem implies

$$
\left\|s_{k} \otimes s_{k}^{\prime}\right\|_{m}=\left\|s_{k}\right\|_{m} \cdot\left\|s_{k}^{\prime}\right\|_{m}=1
$$

Thus

$$
\begin{aligned}
\tau_{m}\left(\left(p, p^{\prime}\right), M \times M^{\prime}\right) & \geq \lim _{k \rightarrow \infty}\left[(-1)^{m\left(n+n^{\prime}\right)^{2} / 2} s_{k} \otimes s_{k}^{\prime}(p) \otimes \overline{s_{k} \otimes s_{k}^{\prime}}(p)\right]^{1 / m} \\
& =\tau_{m}(p, M) \otimes \tau_{m}\left(p^{\prime}, M^{\prime}\right) .
\end{aligned}
$$

On the other hand, for any $S \in \Gamma\left(M \times M^{\prime}, m K_{M \times M^{\prime}}\right)$ satisfying $\|S\|_{m}=1$, we have

$$
\begin{aligned}
{\left[(-1)^{m\left(n+n^{\prime}\right)^{2} / 2} S \otimes \bar{S}\left(p, p^{\prime}\right)\right]^{1 / m} } & \leq \tau_{m}(p, M)(-1)^{n^{\prime 2} / 2}\left\|S\left(\cdot, p^{\prime}\right)\right\|_{m, M}^{2 / m} \\
& \leq \tau_{m}(p, M) \tau_{m}\left(p^{\prime}, M^{\prime}\right)\|S\|_{m, M \times M^{\prime}}^{2 / m} \\
& =\tau_{m}(p, M) \tau_{m}\left(p^{\prime}, M^{\prime}\right) .
\end{aligned}
$$

Since $S$ is arbitrary, the proof is complete.

Suppose now $\tau_{m}$ is nowhere vanishing. For any integer $k \geq 1$, one can define an inner product of $\Gamma\left(M, k K_{M}\right)$ as follows:

$$
\left(s_{1}, s_{2}\right)_{m, k}=\int_{M} \frac{(-1)^{k n^{2} / 2} s_{1} \otimes \bar{s}_{2}}{\tau_{m}^{\otimes(k-1)}} .
$$

Let $\|\cdot\|_{m, k}$ denote the induced norm and $H_{\tau_{m}}^{2}\left(M, k K_{M}\right)$ the Hilbert space of holomorphic sections of $k K_{M}$ which are finite with respect to $\|\cdot\|_{m, k}$. Let 
$s_{1}, s_{2}, \ldots$ be a complete orthonormal basis for $H_{\tau_{m}}^{2}\left(M, k K_{M}\right)$. The associated Bergman kernel is

$$
B_{M}(p)=(-1)^{k n^{2} / 2} \sum_{j} s_{j}(p) \otimes \bar{s}_{j}(p) .
$$

Clearly, $B_{M}$ is independent of the choice of the basis.

Lemma 2.3. $B_{M}$ is invariant under biholomorphic mappings.

Proof. Since $f^{*} \tau_{m}=\tau_{m}$ for a biholomorphic mapping $f$ from $M$ to another complex manifold $N$, one has $\left\|f^{*} s\right\|_{\tau_{m}}=\|s\|_{\tau_{m}}$ for all $s \in H_{\tau_{m}}^{2}\left(N, k K_{N}\right)$. Note that

$$
B_{N}=\sup \left[(-1)^{k n^{2} / 2} s \otimes \bar{s}\right]
$$

where the supremum is taken over all $s \in H_{\tau_{m}}^{2}\left(N, k K_{N}\right)$ with $\|s\|_{\tau_{m}}=1$. The assertion follows by a similar argument to that for Lemma 2.1.

By Lemma 2.2 and a similar argument, we obtain the following product property.

\section{LEMMA 2.4.}

$$
B_{M \times M^{\prime}}=B_{M} \otimes B_{M^{\prime}} .
$$

Definition 2.5. We say that $H_{\tau_{m}}^{2}\left(M, k K_{M}\right)$ is very ample if the following conditions are satisfied:

(B1) Given any $p \in M$, there exists $s \in H_{\tau_{m}}^{2}\left(M, k K_{M}\right)$ such that $s(p)$ $\neq 0$.

(B2) For any holomorphic vector $v$ at $p$, there exists $s \in H_{\tau_{m}}^{2}\left(M, k K_{M}\right)$ such that $s(p)=0$ and $\left.v\left(s^{*}\right)\right|_{p} \neq 0$ where $s=s^{*} d z_{1} \wedge \cdots \wedge d z_{n}$ in local coordinates.

Write

$$
B_{M}(z)=(-1)^{k n^{2} / 2} B_{M}^{*}(z)\left(d z_{1} \wedge \cdots \wedge d z_{n} \wedge d \bar{z}_{1} \wedge \cdots \wedge d \bar{z}_{n}\right)^{\otimes k}
$$

where $B_{M}^{*}(z)$ is a locally defined function. If $B_{M}^{*}$ is nowhere vanishing, we can define a Hermitian form

$$
d s_{M}^{2}=\sum_{j, k} \frac{\partial^{2} \log B_{M}^{*}}{\partial z_{j} \partial \bar{z}_{k}} d z_{j} \otimes d \bar{z}_{k} .
$$

Clearly, $d s_{M}^{2}$ is independent of the choice of the coordinate system, hence is globally defined.

THEOREM 2.6. If $H_{\tau_{m}}^{2}\left(M, k K_{M}\right)$ is very ample, then $d s_{M}^{2}$ defines a Kähler metric which is invariant under biholomorphic mappings.

Proof. Given any $p \in M$ and a holomorphic vector $v$ at $p$, take a complete orthonormal basis $s_{j}, j=1,2, \ldots$, such that

$$
s_{2}(p)=s_{3}(p)=\cdots=0, \quad v\left(s_{2}^{*}\right)(p) \neq 0, \quad v\left(s_{3}^{*}\right)(p)=v\left(s_{4}^{*}\right)(p)=\cdots=0,
$$


where

$$
s_{j}=s_{j}^{*}\left(d z_{1} \wedge \cdots \wedge d z_{n}\right)^{\otimes k}
$$

Thus

$$
B_{M}^{*}(p)=\left|s_{1}(p)\right|^{2}>0, \quad d s_{M}^{2}(p ; v)=\frac{\left|v\left(s_{2}^{*}\right)(p)\right|^{2}}{B_{M}^{*}(p)}>0,
$$

showing the Hermitian positivity of $d s_{M}^{2}$. By Lemma 2.3, one has

$$
B_{M}^{*}(p)=B_{N}^{*}(f(p))\left|J_{f}(p)\right|^{2 k}
$$

where $J_{f}$ is the Jacobian determinant of a biholomorphic mapping $f: M \rightarrow N$, and consequently, $f^{*} d s_{N}^{2}=d s_{M}^{2}$.

Lemmas 2.2 and 2.4 imply the following

TheOREM 2.7. $d s_{M \times M^{\prime}}^{2}=d s_{M}^{2}+d s_{M^{\prime}}^{2}$.

ExAMPLE. Let $M$ be a complex manifold and $\Omega$ a relatively compact domain in $M$. Suppose that $K_{M}$ is ample on some neighborhood $U$ of $\bar{\Omega}$, i.e., there is an integer $m_{0}$ such that $\Gamma\left(U, m_{0} K_{M}\right)$ separates points in $U$ and gives a local coordinate system at each point of $U$. Clearly $d s_{\Omega}^{2}$ exists on $\Omega$ for any $m, k$. In particular, it exists on relatively compact domains in a Stein manifold.

REMARK 2.8. Kobayashi's celebrated criterion for completeness of the standard Bergman metric is still valid for this new invariant metric; the proof is exactly the same as in [8].

Recall that a domain $\Omega$ in a complex manifold $M$ is called locally pseudoconvex if for every $p \in \partial \Omega$, there is a coordinate polydisc $\Delta^{n}$ around $p$ such that $\Omega \cap \Delta^{n}$ is pseudoconvex in the usual sense. We have the following

Bremermann Type Theorem. Let $M$ be a complex manifold and $\Omega$ a relatively compact domain in $M$. Suppose $d s_{\Omega}^{2}$ is a complete Kähler metric on $\Omega$ for some $m, k$. Then $\Omega$ is locally pseudoconvex.

Proof. Since the result is local, we may assume $M=\mathbb{C}^{n}$. If $\Omega$ is not pseudoconvex, then there exists a point $p \in \partial \Omega$ and a neighborhood $U$ of $p$ such that every holomorphic function on $\Omega$ extends to $\Omega \cup U$. By the definition of $\tau_{m}, \tau_{m}^{*}$ extends to a positive continuous function on $\Omega \cap U$, where $\tau_{m}=(-1)^{n^{2} / 2} \tau_{m}^{*} d z_{1} \wedge \cdots \wedge d z_{n} \wedge d \bar{z}_{1} \wedge \cdots \wedge d \bar{z}_{n}$. Since $H_{\tau_{m}}^{2}\left(\Omega, k K_{\Omega}\right)$ is identical with the Hilbert space of holomorphic functions on $\Omega$ which are square-integrable with the weight $k \log \tau_{m}^{*}$, the related Bergman kernel extends to a positive real-analytic function on $\Omega \cup U$, showing $d s_{\Omega}^{2}$ cannot be complete at $p$, a contradiction.

An immediate application is the following 
Siegel Type Theorem. Let $M$ be a complex manifold and $\Omega$ a relatively compact domain in $M$ such that $d s_{\Omega}^{2}$ exists for some $m, k$. If there exists a properly discontinuous group $\Gamma$ of holomorphic transformations of $\Omega$ such that $\Omega / \Gamma$ is compact, then $\Omega$ is locally pseudoconvex.

Proof. The invariant metric $d s_{\Omega}^{2}$ descends to a Kähler metric on the compact manifold $\Omega / \Gamma$, which forces it to be complete on $\Omega$. The above theorem applies.

REMARK 2.9. Lárusson [9] has constructed a class of relatively compact domains in $\mathbb{P}^{n}$ which have compact quotients, but are not locally pseudoconvex.

3. Localization principle. In this section, we give a couple of localization principles.

THEOREM 3.1. Let $M$ be an $n$-dimensional complex manifold and $\Omega$ a relatively compact, locally pseudoconvex domain in $M$. Suppose that $K_{M}$ is ample on some neighborhood $U$ of $\bar{\Omega}$. Then for sufficiently large $m, k$ one has, for every $p \in \partial \Omega$,

$$
C_{1} d s_{\Omega \cap \Delta^{n}}^{2} \leq d s_{\Omega}^{2} \leq C_{2} d s_{\Omega \cap \Delta^{n}}^{2} \quad \text { on } \Omega \cap \Delta_{1 / 2}^{n} .
$$

Here $\Delta^{n}, \Delta_{1 / 2}^{n}$ denote coordinate polydiscs with center $p$ and radius 1 and $1 / 2$ respectively, and $C_{1}, C_{2}$ are positive constants.

Proof. Fix a Kähler metric $\omega$ on $\bar{\Omega}$ (e.g., $\omega$ is generated by $\Gamma\left(U, m_{0} K_{M}\right)$ ). Since $\Omega$ is locally pseudoconvex, there is a constant $C_{3}>0$ such that $-\log \delta_{\Omega}+C_{3} \omega$ is positive in the sense of currents, according to a theorem of Elencwajg [5]. Here $\delta_{\Omega}$ denotes the boundary distance with respect to $\omega$. By a smooth regularization of $-\log \delta_{\Omega}$, there exists a smooth exhaustion function $\psi$ on $\Omega$ such that

$$
\psi-\log \psi+C_{4} \omega
$$

gives a complete Kähler metric on $\Omega$ for sufficiently large $C_{4}$. Given $z_{0} \in$ $\Omega \cap \Delta_{1 / 2}^{n}$, one can choose $s_{1}, \ldots, s_{l} \in \Gamma\left(U, m_{0} K_{M}\right)$ such that

$$
(-1)^{m_{0} n / 2} s_{j} \otimes \bar{s}_{j} \leq C_{5} \omega^{\otimes m_{0}} \quad \text { on } \bar{\Omega}, \quad \sum s_{j} \otimes \bar{s}_{j} \neq 0 \quad \text { on } \Omega \backslash\left\{z_{0}\right\},
$$

and

$$
\begin{gathered}
\partial \bar{\partial} \sum\left|s_{j}^{*}(z)\right|^{2} \geq \partial \bar{\partial}|z|^{2}, \quad \forall z \in \Delta^{n}, \\
\log \sum\left|s_{j}^{*}(z)\right|^{2}=\log |z|^{2}+O(1) \quad \text { near } 0,
\end{gathered}
$$

where $s_{j}=s_{j}^{*}\left(d z_{1} \wedge \cdots \wedge d z_{n}\right)^{\otimes m_{0}}$ on $\Delta^{n}$ and $C_{5}$ is a constant independent of $z_{0}$. Clearly, $\tau_{m_{0}} \geq C \omega$ and its curvature $\Theta_{\tau_{m_{0}}}>0$ (it is not known whether 
$\Theta_{\tau_{m_{0}}}$ dominates $\left.\omega !\right)$. Set

$$
\varphi=(n+1) \log \frac{(-1)^{m_{0} n^{2} / 2} \sum s_{j} \otimes \bar{s}_{j}}{\tau_{m_{0}}^{\otimes m_{0}}} .
$$

Clearly, $\varphi$ is bounded above by a constant. Set $m_{1}=(n+1) m_{0}$ and define a singular Hermitian metric $h^{-1}$ on $m_{1} K_{\Omega}$ by

$$
h=\tau_{m_{0}}^{\otimes m_{1}} e^{\varphi} \text {. }
$$

Then $h^{-1}$ is smooth and positive outside $z_{0}$ and $\Theta_{h^{-1}}$ dominates the Euclidean metric on $\Delta^{n}$. Let $\chi$ be a smooth cut-off function such that $\left.\chi\right|_{(-\infty, 2 / 3]}$ $=1$ and $\left.\chi\right|_{[1, \infty)}=0$. For any local section $s \in H_{\tau_{m_{0}}}^{2}\left(\Omega \cap \Delta^{n},\left(m_{1}+1\right) K_{\Delta^{n}}\right)$, we can solve $\bar{\partial} u=s \otimes \bar{\partial} \chi(|z|)$ by the standard $L^{2}$-theory on complete Kähler manifolds (compare [2], [13]) together with the estimate

$$
\int_{\Omega} \frac{(-1)^{\left(m_{1}+1\right) n^{2} / 2} u \otimes \bar{u}}{h} \leq C_{6} \int_{\Omega \cap \Delta^{n}} \frac{(-1)^{\left(m_{1}+1\right) n^{2} / 2} s \otimes \bar{s}}{\tau_{m_{0}}^{\otimes(n+1)}} .
$$

Since $\varphi$ is bounded above by a constant, we conclude that $S:=\chi(|z|) s-u \in$ $\Gamma\left(\Omega,\left(m_{1}+1\right) K_{\Omega}\right)$ is such that

$$
\int_{\Omega} \frac{(-1)^{\left(m_{1}+1\right) n^{2} / 2} S \otimes \bar{S}}{\tau_{m_{0}}^{\otimes m_{1}}} \leq C_{7} \int_{\Omega \cap \Delta^{n}} \frac{(-1)^{\left(m_{1}+1\right) n^{2} / 2} s \otimes \bar{s}}{\tau_{m_{0}}^{\otimes m_{1}}}
$$

and $S^{*}\left(z_{0}\right)=s^{*}\left(z_{0}\right),\left(\partial S^{*} / \partial z_{j}\right)\left(z_{0}\right)=\left(\partial s^{*} / \partial z_{j}\right)\left(z_{0}\right)$ for $j=1, \ldots, n$, where $S^{*}, s^{*}$ are local representations of $S, s$. Thus the assertion follows.

Question. Is $d s_{\Omega}^{2}$ complete under the hypothesis of Theorem 3.1?

Next we give a localization principle for holomorphic fiber bundles.

TheOREM 3.2. Let $\pi: M \rightarrow B$ be a holomorphic fiber bundle such that the base $B$ is a compact complex manifold with ample canonical bundle and the typical fiber $F$ is a bounded pseudoconvex domain in $\mathbb{C}^{n}$. Then for sufficiently large $m, k$ one has, for every $p \in B$ and a coordinate polydisc $\Delta^{l}$ at $p$,

$$
C_{1} d s_{\pi^{-1}\left(\Delta^{l}\right)}^{2} \leq d s_{M}^{2} \leq C_{2} d s_{\pi^{-1}\left(\Delta^{l}\right)}^{2} \quad \text { on } \pi^{-1}\left(\Delta_{1 / 2}^{l}\right) .
$$

Proof. Observe that $M$ carries a complete Kähler metric: as $F$ is a bounded domain of holomorphy, it admits a complete Kähler-Einstein metric $\omega_{F}$ which is invariant under the group of holomorphic transformations of $F$; one simply chooses the complete metric to be $\omega_{F}+\omega_{B}$ with $\omega_{B}$ an arbitrary Kähler metric on $B$. Similarly, $\tau_{m}(F)$ is also invariant under the group of holomorphic transformations of $F$, hence one has the decomposition $\tau_{m}(M)=\tau_{m}(B) \otimes \tau_{m}(F)$ for any $m \geq 1$. A similar application of the $L^{2}$-theory on complete Kähler manifolds yields the assertion. 
4. More examples on existence. The localization principle provides enough examples for the existence of the invariant metric. In this section, we give more examples.

THEOREM 4.1. Let $M$ be a complex manifold with trivial canonical bundle (e.g. a torus) and $\Omega$ a relatively compact Stein domain in M. Suppose the following conditions are satisfied:

(i) There exists a smooth, strictly plurisubharmonic exhaustion function $\psi$ on $\Omega$ such that $C_{1} \log 1 / \delta_{\Omega} \leq \psi \leq C_{2} \log 1 / \delta_{\Omega}$ and $\partial \bar{\partial} \psi \geq$ $C_{3} \omega$, where $\omega$ is a fixed Hermitian metric on $M$ and $\delta_{\Omega}$ denotes the distance to $\partial \Omega$ with respect to $\omega$.

(ii) There is a number $\alpha>0$ such that $\int_{\Omega} \delta_{\Omega}^{-\alpha} d V_{\omega}<\infty$, where $d V_{\omega}$ denotes the volume form with respect to $\omega$ (e.g. $\partial \Omega$ is Lipschitz).

Then $d s_{\Omega}^{2}$ exists on $\Omega$ for sufficiently large $m, k$.

Theorem 4.1 will be proved by a number of lemmas.

Lemma 4.2. For $a>0$, we define

$$
\mathcal{O}_{a}^{2}(\Omega):=\left\{f \in \mathcal{O}(\Omega): \int_{\Omega}|f|^{2} \delta_{\Omega}^{a} d V_{\omega}<\infty\right\}
$$

where $\mathcal{O}(\Omega)$ is the sheaf of holomorphic functions on $\Omega$. Then for sufficiently large a, $\mathcal{O}_{a}^{2}(\Omega)$ separates points and gives local coordinate systems in $\Omega$. Furthermore, there exist positive numbers $a_{1}, C$ such that

$$
\sup _{f \in \mathcal{O}_{a}^{2}(\Omega)} \frac{|f(p)|^{2}}{\int_{\Omega}|f|^{2} \delta_{\Omega}^{a} d V_{\omega}} \geq C \delta_{\Omega}(p)^{-a_{1}}, \quad \forall p \in \Omega .
$$

Proof. By compactness of $\bar{\Omega}$, for every $p \in \Omega$ we have a coordinate polydisc $\Delta^{n}$ around $p$ such that $\omega$ is equivalent to the Euclidean metric on $\Delta^{n}$ with the implicit constants independent of $p$. By hypothesis (i), we have

$$
\partial \bar{\partial}(C \psi+2(n+1) \chi(|z|) \log |z|)+\operatorname{Ricci}(\omega)>\omega
$$

for sufficiently large $C$. Equipping the anti-canonical bundle $K_{M}^{\otimes(-1)}$ with the Hermitian metric

$$
h=\omega^{n} e^{-C \psi-2(n+1) \chi(|z|) \log |z|,}
$$

and applying the $L^{2}$-existence theorem [4] for $K_{M}^{\otimes(-1)}$-valued $(n, 1)$-forms with respect to not necessarily complete metrics in a similar way as above, we conclude that those $f$ in $\mathcal{O}(\Omega)$ satisfying

$$
\int_{\Omega}|f|^{2} e^{-C \psi} d V_{\omega}<\infty
$$

separate points and give local coordinate systems in $\Omega$. The first assertion follows immediately from $\psi \asymp \log 1 / \delta_{\Omega}$. To show the second assertion, we 
see that the above argument in fact gives a localization principle for the Bergman kernel of the Hilbert space of holomorphic functions on $\Omega$ which are square-integrable with weight $C \psi$, thus one can reduce to the case of bounded pseudoconvex domains in $\mathbb{C}^{n}$. On the other hand, Demailly's theorem [3] implies that this Bergman kernel always dominates $e^{C \psi}$. This completes the proof.

LEMMA 4.3.

$$
f \in \mathcal{O}_{a}^{2}(\Omega) \Rightarrow \sup _{p \in \Omega}|f(p)|^{2} \delta_{\Omega}(p)^{2 n+a}<\infty .
$$

Proof. Since the assertion is local, we may assume $\Omega$ is a bounded domain in $\mathbb{C}^{n}$ and $\omega$ is the Euclidean metric. By the sub-mean-value principle, we have, for arbitrary $p \in \Omega$,

$$
|f(p)|^{2} \leq \frac{1}{\operatorname{Vol}\left\{B\left(p ; \delta_{\Omega}(p)\right)\right\}} \int_{\left.B\left(p ; \delta_{\Omega}(p)\right)\right)}|f|^{2} d V \leq C_{n} \delta_{\Omega}(p)^{-2 n-a} \int_{\Omega}|f|^{2} \delta_{\Omega}^{a} d V
$$

where $B(p ; r)$ denotes the Euclidean ball with center $p$ and radius $r$. We are done.

Let us fix a nowhere vanishing holomorphic section $s_{0}$ of $K_{M}$.

Lemma 4.4. If $m \gg a$, then for any $f \in \mathcal{O}_{a}^{2}(\Omega)$ we have

$$
s:=f s_{0}^{\otimes m} \in \Gamma\left(\Omega, m K_{\Omega}\right)
$$

and

$$
\|s\|_{m}=\left\{\int_{\Omega}\left[(-1)^{m n^{2} / 2} s \otimes \bar{s}\right]^{1 / m}\right\}^{m / 2}<\infty .
$$

Furthermore, $\tau_{m}$ dominates $\delta_{\Omega}^{-a_{1} / m}$ where $a_{1}$ is as in Lemma 4.2.

Proof. By Lemma 4.3, there is a constant $C>0$ depending only on the $L^{2}$ norm of $f$ in $\mathcal{O}_{a}^{2}(\Omega)$ such that $|f|^{2} \leq C \delta_{\Omega}^{-2 n-a}$ on $\Omega$. By hypothesis (ii) we see that the conclusion holds when

$$
m \geq \frac{2 n+a}{\alpha} \text {. }
$$

The second assertion follows from Lemma 2.2.

Proof of Theorem 4.1. Fix $m \gg a \gg 1$ such that the conclusions of Lemmas 4.2 and 4.4 hold. By Lemma 4.4, we claim that for any $f \in \mathcal{O}_{a}^{2}(\Omega)$,

$$
s:=f s_{0}^{\otimes k} \in H_{\tau_{m}}^{2}\left(\Omega, k K_{\Omega}\right)
$$

provided

$$
\frac{(k-1) a_{1}}{m} \geq a \quad \text { or } \quad k \geq \frac{a m}{a_{1}}+1 .
$$

Therefore, Lemma 4.2 implies the existence of $d s_{\Omega}^{2}$.

Conjecture. Hypothesis (ii) in Theorem 4.1 is not necessary. 
REMARK 4.5. It is also interesting to consider relatively compact domains in a complex manifold whose canonical bundle is neither positive nor trivial. For instance, Nemirovskiu [12] has constructed a smooth Levi-flat Stein domain in the product of a compact Riemann surface and a onedimensional torus which is biholomorphically equivalent to the product of a punctured Riemann surface (remove finitely many points from the original Riemann surface) and an annulus. By Theorems 2.7 and 3.1, this domain carries an invariant metric if the genus of the Riemann surface is larger than 1.

5. Asymptotic behavior. In this section, we study the asymptotic behavior of $d s_{\Omega}^{2}$ when $\partial \Omega$ is relatively simple, namely, we consider the domain $\Omega=M-D$ where $M$ is a compact complex manifold and $D$ is an effective divisor with only simple normal crossings.

THEOREM 5.1. Let $M$ be a compact complex manifold with ample canonical line bundle and $D$ an effective divisor with only simple normal crossings. Then for sufficiently large $m, k, d s_{\Omega}^{2}$ is a complete Kähler metric on $\Omega$. Furthermore, the distance is equivalent to $-\log \delta_{\Omega}$ where $\delta_{\Omega}$ is the boundary distance with respect to a Hermitian metric on $M$.

REMARK 5.2. If in addition $D$ is ample, then $\Omega$ admits an invariant Kähler-Einstein metric whose distance is equivalent to $\log \left|\log \delta_{\Omega}\right|$ (cf. [7]). Thus $d s_{\Omega}^{2}$ is not equivalent to the Kähler-Einstein metric.

Proof of Theorem 5.1. First fix a positive smooth Hermitian metric $h$ of $K_{M}$ such that the curvature $\omega:=\Theta(h)$ gives a Kähler metric on $M$. Write $D=D_{1}+\cdots+D_{N}$ where the irreducible components $D_{i}$ are smooth and intersect transversely. Let $\sigma_{i}$ be a holomorphic section of $\left[D_{i}\right]$ defining $D_{i}$ and set $\sigma=\sigma_{1} \otimes \cdots \otimes \sigma_{N}$. Let $\|\cdot\|$ denote the norm with respect to a Hermitian metric for $\left[D_{i}\right]$ and also the norm for the product $[D]=\left[D_{1}\right] \otimes \cdots \otimes\left[D_{N}\right]$ associated to the induced metric. Assume $\|\sigma\|<1$ for simplicity. It is easy to verify that

$$
C \omega-\log (-\log \|\sigma\|)
$$

defines a complete Kähler metric on $\Omega$ for large $C>0$. Setting

$$
H_{h}^{2}\left(\Omega, m K_{\Omega}\right)=\left\{s \in \Gamma\left(\Omega, m K_{\Omega}\right):\|s\|_{h, m}^{2}=\int_{\Omega} \frac{(-1)^{m n^{2} / 2} s \otimes \bar{s}}{h^{\otimes(m-1)}\|\sigma\|^{-1 / 2}}<\infty\right\} .
$$

Let $B_{\Omega, h, m}$ denote the Bergman kernel of $H_{h}^{2}\left(\Omega, m K_{\Omega}\right)$, i.e.,

$$
B_{\Omega, h, m}=\sup _{\|s\|_{h, m}=1}\left[(-1)^{m n^{2} / 2} s \otimes \bar{s}\right] .
$$

LEMMA 5.3. There is an integer $m_{0}>1$ such that for all $m \geq m_{0}$, $B_{\Omega, h, m}$ is a smooth Hermitian metric on $m K_{\Omega}$ such that

$$
C_{m}^{\prime}\|\sigma\|^{-2} \leq B_{\Omega, h, m} / h^{\otimes m} \leq C_{m}^{\prime \prime}\|\sigma\|^{-2} .
$$


Proof. By compactness of $M$, there is an integer $m_{0}>0$ such that for any $m \geq m_{0}$ and any $p \in \Omega$, there is a coordinate polydisc $\left(\Delta^{n}, z\right)$ with $z(p)=0$ such that the singular Hermitian metric

$$
h_{m-1}:=h^{\otimes(m-1)}\|\sigma\|^{-1 / 2} e^{-2(n+1) \chi(|z|) \log |z|}
$$

is smooth and positive on $\Omega-\{p\}$ and dominates $\partial \bar{\partial}|z|^{2}$ on $\Omega \cap \Delta^{n}$. A standard application of the $L^{2}$-theory shows that the localization principle holds for $B_{\Omega, h, m}$. Note that for any $p \in D$, there is a coordinate polydisc $\Delta^{n}$ centered at $p$ such that

$$
\Omega \cap \Delta^{n}=\left(\Delta^{*}\right)^{k} \times \Delta^{n-k}
$$

for some $1 \leq k \leq N$ where $\Delta^{*}$ denotes the punctured disc. It is clear that

$$
\begin{aligned}
s=s^{*}\left(d z_{1} \wedge \cdots \wedge d z_{n}\right)^{\otimes m} & \in H_{h}^{2}\left(\Omega \cap \Delta^{n}, m K_{\Omega}\right) \\
& \Leftrightarrow c_{\alpha_{1} \cdots \alpha_{n}}=0 \text { if } \alpha_{i}<-1 \text { for some } 1 \leq i \leq k,
\end{aligned}
$$

where

$$
s^{*}=\sum c_{\alpha_{1} \cdots \alpha_{n}} z_{1}^{\alpha_{1}} \cdots z_{n}^{\alpha_{n}}
$$

is the Laurent expansion. By the localization principle, the assertion follows.

Now fix such an $m_{0}>1$. Let $\tau_{m_{0}}$ be defined as in Section 2. As $\|\sigma\|^{-2 / m_{0}}$ is integrable on $\Omega$, Lemma 5.3 implies $\tau_{m_{0}} / h \geq C\|\sigma\|^{-2 / m_{0}}$. Let $\Delta^{n}$ be a coordinate polydisc around a boundary point $p$ as in (1) and let $z^{*} \in \Delta^{n} \cap \Omega$ be any point. Take $s \in \Gamma\left(\Omega, m_{0} K_{\Omega}\right)$ such that

$$
\|s\|_{m_{0}}=\left\{\int_{\Omega}\left[(-1)^{m_{0} n^{2} / 2} s \otimes \bar{s}\right]^{1 / m_{0}}\right\}^{m_{0} / 2}=1
$$

and

$$
\tau_{m_{0}}\left(z^{*}\right) \leq 2\left[(-1)^{m_{0} n^{2} / 2} s \otimes \bar{s}\left(z^{*}\right)\right]^{1 / m_{0}} .
$$

Considering the Laurent expansion of $s^{*}$, we conclude that there exist positive integers $\lambda_{i}, i=1, \ldots, k$, such that

$$
\tau_{m_{0}}\left(z^{*}\right) \asymp \prod_{1 \leq i \leq k}\left\|\sigma_{i}\right\|^{-2 \lambda_{i} / m_{0}}\left(z^{*}\right) .
$$

LEMmA 5.4. There exists an integer $k_{0} \geq 2$ such that $H_{\tau_{m_{0}}}^{2}\left(\Omega, k_{0} K_{\Omega}\right)$ is very ample and the ratio of its Bergman kernel and $h^{\otimes k_{0}}$ dominates $\|\sigma\|^{-2}$.

Proof. The localization principle also holds for the Bergman kernel of the Hilbert space

$$
\mathcal{H}_{k}:=\left\{s \in \Gamma\left(\Omega, k K_{\Omega}\right): \int_{\Omega} \frac{(-1)^{k n^{2} / 2} s \otimes \bar{s}}{\tau_{m_{0}} \otimes h^{\otimes(k-1)}}<\infty\right\}
$$

provided $k$ sufficiently large, and its Bergman kernel dominates $\|\sigma\|^{-2}$ according to (2). Since $\mathcal{H}_{k} \subset H_{\tau_{m_{0}}}^{2}\left(\Omega, k K_{\Omega}\right)$, we are done. 
End of proof of Theorem 5.1. Lemma 5.4 implies the existence of $d s_{\Omega}^{2}$. Consider again the coordinate polydisc $\Delta^{n}$ chosen as in (1). Given any $z^{*} \in \Omega \cap \Delta^{n}$, suppose that the Bergman kernel at $z^{*}$ equals $\left[(-1)^{k_{0} n^{2} / 2} s \otimes\right.$ $\left.\bar{s}\left(z^{*}\right)\right]^{1 / k_{0}}$ for some $s \in H_{\tau_{m_{0}}}^{2}\left(\Omega, k_{0} K_{\Omega}\right)$ with unit norm. As above, we conclude from (2) and Lemma 5.4 that there are positive integers $l_{1}, \ldots, l_{k}$ such that

$$
\begin{aligned}
\left|s^{*}\left(z^{*}\right)\right| & \asymp \prod_{1 \leq i \leq k}\left\|\sigma_{i}\right\|^{-l_{i}}\left(z^{*}\right), \\
\left|\left(\partial s^{*} / \partial z_{i}\right)\left(z^{*}\right)\right| & \asymp\left\|\sigma_{1}\right\|^{-l_{1}} \cdots\left\|\sigma_{i}\right\|^{-l_{i}-1} \cdots\left\|\sigma_{k}\right\|^{-l_{k}}\left(z^{*}\right) \quad \forall 1 \leq i \leq k .
\end{aligned}
$$

The assertion follows immediately from the extremal property of the invariant metric.

\section{References}

[1] S. Y. Cheng and S. T. Yau, On the existence of a complete Kähler metric on noncompact complex manifolds and the regularity of Fefferman's equation, Comm. Pure Appl. Math. 33 (1980), 507-544.

[2] J.-P. Demailly, Estimations $L^{2}$ pour l'opérateur $\bar{\partial}$ d'un fibré vectoriel holomorphe semi-positif au-dessus d'une variété kählérienne complète, Ann. Sci. École Norm. Sup. 15 (1982), 457-511.

[3] -, Regularization of closed positive currents and intersection theory, J. Algebraic Geom. 1 (1992), 361-409.

[4] -, Multiplier Ideal Sheaves and Analytic Methods in Algebraic Geometry, ICTP lectures, Trieste, 2000.

[5] G. Elencwajg, Pseudo-convexité locale dans les variétés kählériennes, Ann. Inst. Fourier (Grenoble) 25 (1975), no. 2, 295-314.

[6] M. Jarnicki and P. Pflug, Invariant Distances and Metrics in Complex Analysis, de Gruyter Exp. Math. 9, de Gruyter, Berlin, 1993.

[7] R. Kobayashi, Kähler-Einstein metric on an open algebraic manifold, Osaka J. Math. 21 (1984), 399-418.

[8] S. Kobayashi, Geometry of bounded domains, Trans. Amer. Math. Soc. 92 (1959), 267-290.

[9] F. Lárusson, Compact quotients of large domains in complex projective space, Ann. Inst. Fourier (Grenoble) 48 (1998), 223-246.

[10] N. Mok and S. T. Yau, Completeness of the Kähler-Einstein metric on bounded domains and characterization of domains of holomorphy by curvature conditions, in: Proc. Sympos. Pure Math. 39, Amer. Math. Soc., 1983, 41-60.

[11] M. S. Narasimhan and R. R. Simha, Manifolds with ample canonical class, Invent. Math. 5 (1968), 120-128.

[12] S. Nemirovskiı̌, Stein domains with Levi-flat boundaries on compact complex surfaces, Math. Notes 66 (1999), 522-525. 
[13] T. Ohsawa, On complete Kähler domains with $C^{1}$-boundary, Publ. RIMS Kyoto Univ. 16 (1980), 929-940.

[14] H. Tsuji, Refined semipositivity and moduli of canonical models, preprint, 2005.

Department of Mathematics

Tongji University

Shanghai 200092, P.R. China

E-mail: boychen@mail.tongji.edu.cn

Received 10.10.2006 